\title{
Development of a novel lipid metabolism-based risk score model in hepatocellular carcinoma patients
}

\author{
Wenjie Wang ${ }^{1 \dagger}$, Chen Zhang ${ }^{1 \dagger}$, Qihong Yu ${ }^{1,2,3}$, Xichuan Zheng ${ }^{1}$, Chuanzheng Yin ${ }^{1}$, Xueke Yan ${ }^{1}$, Gang Liu ${ }^{4}$ \\ and Zifang Song ${ }^{1 *}$
}

\begin{abstract}
Background: Liver cancer is one of the most common malignancies worldwide. HCC (hepatocellular carcinoma) is the predominant pathological type of liver cancer, accounting for approximately $75-85 \%$ of all liver cancers. Lipid metabolic reprogramming has emerged as an important feature of HCC. However, the influence of lipid metabolismrelated gene expression in HCC patient prognosis remains unknown. In this study, we performed a comprehensive analysis of HCC gene expression data from TCGA (The Cancer Genome Atlas) to acquire further insight into the role of lipid metabolism-related genes in HCC patient prognosis.

Methods: We analyzed the mRNA expression profiles of 424 HCC patients from the TCGA database. GSEA(Gene Set Enrichment Analysis) was performed to identify lipid metabolism-related gene sets associated with HCC. We performed univariate Cox regression and LASSO(least absolute shrinkage and selection operator) regression analyses to identify genes with prognostic value and develop a prognostic model, which was tested in a validation cohort. We performed Kaplan-Meier survival and ROC (receiver operating characteristic) analyses to evaluate the performance of the model.

Results: We identified three lipid metabolism-related genes (ME1, MED10, MED22) with prognostic value in HCC and used them to calculate a risk score for each HCC patient. High-risk HCC patients exhibited a significantly lower survival rate than low-risk patients. Multivariate Cox regression analysis revealed that the 3 -gene signature was an independent prognostic factor in HCC. Furthermore, the signature provided a highly accurate prediction of HCC patient prognosis.

Conclusions: We identified three lipid-metabolism-related genes that are upregulated in HCC tissues and established a 3-gene signature-based risk model that can accurately predict HCC patient prognosis. Our findings support the strong links between lipid metabolism and HCC and may facilitate the development of new metabolism-targeted treatment approaches for HCC.
\end{abstract}

Keywords: Lipid metabolism, Hepatocellular carcinoma, Predicting model

\section{*Correspondence: zsong@hust.edu.cn}

'Wenjie Wang and Chen Zhang contributed equally to this work

1 Department of Hepatobiliary Surgery, Union Hospital, Tongji Medical College, Huazhong University of Science and Technology, 1277 Jiefang Avenue, Wuhan 430022, China

Full list of author information is available at the end of the article

\section{Background}

Liver cancer is one of the most common malignancies worldwide [1], with HCC being the most predominant pathological types of primary liver cancer [2]. The most common treatment methods for HCC are surgical resection and systemic, comprehensive treatment [3]. However, $\mathrm{HCC}$ is often diagnosed at late stages, leading

(c) The Author(s) 2021. This article is licensed under a Creative Commons Attribution 4.0 International License, which permits use, sharing, adaptation, distribution and reproduction in any medium or format, as long as you give appropriate credit to the original author(s) and the source, provide a link to the Creative Commons licence, and indicate if changes were made. The images or other third party material in this article are included in the article's Creative Commons licence, unless indicated otherwise in a credit line to the material. If material is not included in the article's Creative Commons licence and your intended use is not permitted by statutory regulation or exceeds the permitted use, you will need to obtain permission directly from the copyright holder. To view a copy of this licence, visit http://creativecommons.org/licenses/by/4.0/. The Creative Commons Public Domain Dedication waiver (http://creativecommons.org/publicdomain/zero/1.0/) applies to the data made available in this article, unless otherwise stated in a credit line to the data. 
to high rates of recurrence and metastasis after surgical resection, resistance to chemotherapy, and poor 5-year overall survival rate $(<12 \%)[4,5]$. Common traditional clinicopathological characteristics, such as age, gender, and incision margin, cannot accurately predict the prognosis of HCC patients [6]. Therefore, the development of novel effective treatment methods and the identification of robust prognostic biomarkers are urgently needed.

Metabolic reprogramming is widely accepted as a hallmark of cancer. It involves aberrant alterations in various key metabolic pathways, including glycolysis and fatty acid, glutamine, and cholesterol metabolic pathways [7]. Metabolic reprogramming is essential for cancer cells to meet their high energy demands due to their rapid growth, as well as to adapt to changes in the tumor microenvironment [8]. Metabolic reprogramming is particularly important in liver cancer [9]. The liver is essential in maintaining the energy balance in our body and regulating lipid metabolism. Mounting evidence suggests that lipid metabolic reprogramming is strongly linked to cancer development and progression [10] and that alterations in lipid metabolism are common in HCC [11]. Thus, modulating lipid metabolic pathways may represent a promising treatment approach for HCC. Nevertheless, the effect of lipid metabolism-related gene expression in HCC patient prognosis remains unclear.

In this study, we performed GSEA to identify lipid metabolism gene sets that are significantly enriched in HCC patients. We also explored the role of lipid metabolism-related genes in HCC patient prognosis by combining mRNA expression profiles and clinical data from TCGA database. We identified three lipid metabolismrelated genes that accurately predicted HCC patient prognosis. The findings presented herein strongly support that lipid metabolism has a promising prognostic and therapeutic value in HCC.

\section{Methods}

\section{Gene expression data acquisition}

Gene expression data, gene methylation profiles, and clinicopathological characteristics of HCC patients were obtained from TCGA [12]. The clinical information included survival time, survival status, gender, age, and tumor grade and stage; the samples of patients with missing clinical information were excluded. Data from 424 patients were analyzed in this study, and their clinical information was examined. Gene mutations in HCC patients were identified using cBioPortal (https://www. cbioportal.org/) [13].

\section{Human protein atlas (HPA)}

The human protein atlas database (https://www.prote inatlas.org/) was used to obtain immunohistochemistry staining data. These data were used to assess the levels of different proteins in normal and tumor tissues.

\section{Enrichment analysis}

GSEA was performed to identify gene sets significantly enriched in HCC patients [14]. The analysis was conducted using the GSEA v. 4.0.2 software from Broad Institute (http://www.broadinstitute.org/gsea). GSEA was carried out using a weighting method, and the number of random combinations was set to 1000; the minimum number of excluded genes was set to 1 . The remaining parameters were set to default. False discovery rate (FDR) q-value $<0.05$ was considered significant.

Functional enrichment analysis was performed using DAVID (The Database for Annotation, Visualization, and Integrated Discovery; https://david.ncifcrf.gov/) [15]. Enriched functions were visualized using SangerBox (http://sangerbox.com/Tool).

\section{Statistical analysis}

Bioinformatic analyses were conducted using $\mathrm{R}$ version 3.6.1. We used the Wilcoxon rank-sum test to identify differentially expressed genes between $\mathrm{HCC}$ and normal tissues. Univariate Cox regression analysis was performed using the $\mathrm{R}$ survival package to identify genes with significant prognostic value $(p<0.05)$. LASSO regression was implemented by using the "glmnet" package to build a prognostic model. Combining regression coefficients and mRNA expression profiles, we established a formula to calculate a prognostic risk score: risk score $=\sum($ Coefi $\times \mathrm{Xi})$. Coefi is the regression coefficient, and $\mathrm{Xi}$ represents the corresponding mRNA expression. A risk curve was plotted using $\mathrm{R}$ to evaluate the prognostic model. The differences in the overall survival of patients were assessed by using Kaplan-Meier analysis and log-rank method. The predictive performance of the model was evaluated using ROC analysis and the AUC (area under the curve) values.

\section{Results \\ Identification of the lipid metabolism-related gene sets in $\mathrm{HCC}$}

First, we identified nine gene sets associated with lipid metabolism by searching the keyword "lipid metabolism" in the MSigDB database. Subsequently, we assess for enrichment of these gene sets in HCC. GSEA results indicated that only two lipid metabolism-related gene sets were significantly enriched in HCC samples: "Reactome regulation of lipid metabolism by pparalpha" and "SA/PTEN pathway" ( $n=163$ genes; Fig. 1a). To investigate the expression level of these genes in HCC, we downloaded RNA-seq data from TCGA. We identified 35 lipid metabolism-related genes differently expressed 
between normal and tumor samples $(p<0.05,|\log \mathrm{FC}|>1$; Table 1). Next, we performed GO (gene ontology) enrichment analysis to get further insight into the function of these genes and confirmed their role in lipid metabolism (Fig. 1b).

\section{Identification of lipid metabolism-related genes with prognostic value in $\mathrm{HCC}$}

To assess the relationship between the 35 lipid metabolism-related genes and HCC patients' overall survival, we performed univariate Cox regression analysis. We identified 11 genes that were significantly associated with patient prognosis (Table 2). Ten of these 11 genes were associated with poor prognosis (hazard ratio $[\mathrm{HR}]>1$ ), and one gene (CYP4A11) predicted favorable outcomes $(\mathrm{HR}<1)$. To selected the genes based on the minimum criterion, we conducted LASSO regression (Fig. 1c), which allows for the handling of variables even when there is collinearity between data [16]. Three lipid metabolism-related genes (ME1, MED10, MED22) were associated with patient survival.

\section{ME1, MED10, andMED22expression levels are elevated in HCC tissues}

We assessed the expression levels of $M E 1, M E D 10$, and $M E D 22$ in $\mathrm{HCC}$ and normal tissues using data from TCGA and HPA and found that they were highly expressed in HCC tissues, both at the mRNA and protein levels (Fig. 2a, b). Based on the median expression of each gene, HCC patients were stratified into high-expression and low-expression groups. Survival analysis revealed that the expression level of each gene was associated with $\mathrm{HCC}$ patient survival (Fig. 2c).

To investigate the possible mechanism underlying $M E 1, M E D 10$, and MED22 upregulation in HCC, we analyzed the methylation levels of these genes in HCC patients. The mRNA levels of ME1, MED10, and MED22 were negatively correlated with their overall methylation levels (Fig. 2d), suggesting that their upregulation in HCC tissues might be due to a reduction in methylation levels. Furthermore, mutation analysis in HCC samples revealed that $0.8 \%, 2.8 \%$, and $1.7 \%$ of patients had mutations in $M E D 22, M E 1$, and $M E D 10$, respectively (Fig. 2e). Interestingly, most of the genetic alterations in these genes involved amplifications and deep deletions, suggested a potential role of gene mutations in ME1, MED10, and MED22 upregulation in $\mathrm{HCC}$.

\section{Establishment and validation of a prognostic model}

We randomly divided $370 \mathrm{HCC}$ patients into a training set $(n=185)$ and a validation set $(n=185)$. Combining the regression coefficients of the three genes and their mRNA levels, we calculated the corresponding risk score for each patient [Risk score $=(0.0144 \times$ Expression of ME1 $)+(0.0030 \times$ Expression of MED10 $)+(0.0763$ $\times$ Expression of MED22)]. To construct a prognostic model based on these genes, we divided the patients in the training set into high-risk and low-risk groups according to their risk score (the median value was used as a cut-off; Fig. 3a). Interestingly, a high-risk score was associated with shorter survival time and increased mortality rate (Fig. 3b). Consistently, Kaplan-Meier analysis revealed a significant difference in overall survival between high-risk and low-risk patients, with a 5-year survival rate of 0.3 in the high-risk group and 0.6 in the low-risk group (Fig. 3c). ROC curve showed an AUC value of 0.74 (Fig. 3d), confirming the high prognostic value of our 3-gene signature in $\mathrm{HCC}$.

We also verified the relation between clinical features and risk score by doing chi-square test (Table 3 ). The results revealed that age, grade and relative family cancer history significantly concerned the risk score of HCC patients.

Subsequently, we tested our prognostic model in the validation set. Consistent with the findings in the training set, patients in the high-risk group had worse outcomes than those in the low-risk group (Fig. 3c). In the validation set, the prognostic model provided an AUC value of 0.762 (Fig. 3d), confirming that the 3-gene signature can accurately predict $\mathrm{HCC}$ patient prognosis.

\section{The 3-gene signature is an independent prognostic factor in $\mathrm{HCC}$}

To explore whether the risk score based on these three genes could be used as an independent prognostic factor, we performed univariate and multivariate cox regression analyses and found that the risk score was significantly associated with HCC patient prognosis (Fig. 4a, b). We also found that the tumor stage was an independent prognostic factor for poor prognosis.

(See figure on next page.)

Fig. 1 Enrichment analysis and LASSO regression analysis in HCC. a Gene set enrichment analysis of two lipid metabolism-related gene sets in HCC. b GO enrichment analysis of differentially expressed lipid metabolism-related genes in biological process. c Plots of regression coefficient using the LASSO model. On the left is the variation of LASSO coefficient with lambda for the 11 mRNAs from univariate Cox regression analysis. The first dotted line on the right represents the minimum values for logarithm Lambda according to the regression model 
a
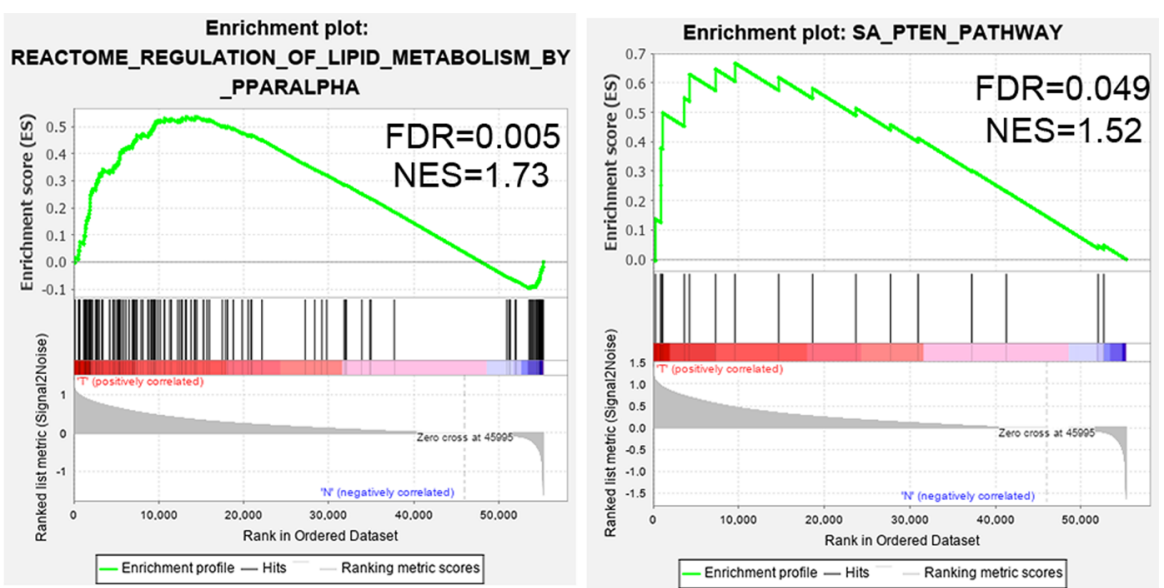

b

positive regulation of transcription from RNA polymerase II promote

regulation of transcription from RNA polymerase II promoter positive regulation of transcription, DNA-templated transcription, DNA-templated negative regulation of transcription from RNA polymerase II promoter protein ubiquitination lipid metabolic process heart development response to low-density lipoprotein particle positive regulation of cholesterol storage positive regulation of phagocytosis, engulfment response to lipid lipoprotein transport intracellular steroid hormone receptor signaling pathway response to vitamin $\mathrm{A}$ Fc-epsilon receptor signaling pathway arachidonic acid metabolic process

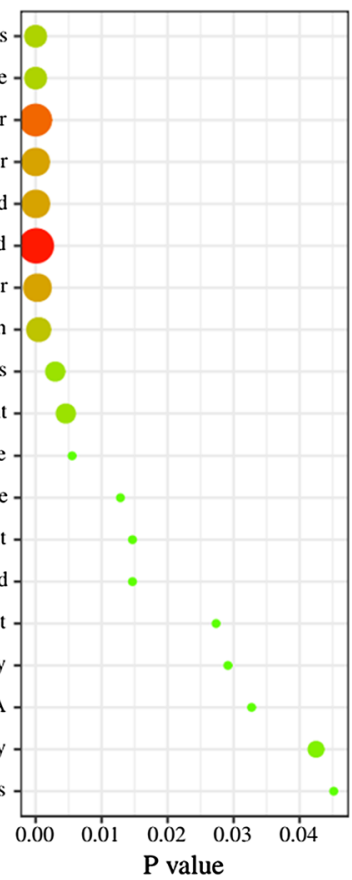

Gene Raito 0.20 0.15 0.10 0.05

Gene count

- 2.5

5.0

7.5

- 10.0

( 12.5

C
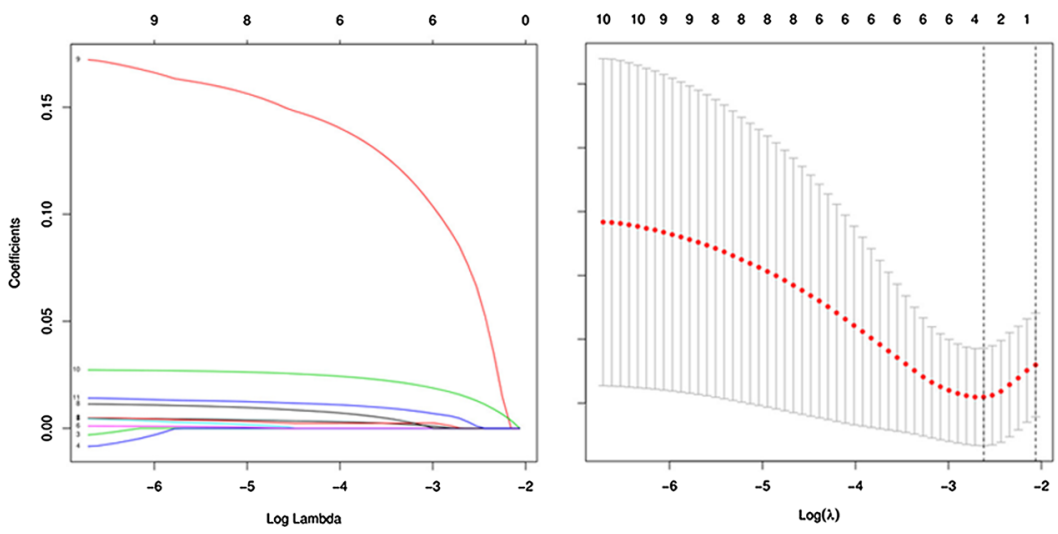
Table 1 The detailed information of 35 mRNAs significantly up regulated in HCC patients

\begin{tabular}{|c|c|c|}
\hline \multicolumn{3}{|c|}{ Up-regulated gene set } \\
\hline mRNA & Ensembl ID & Description \\
\hline TIAM2 & ENSG00000146426 & TIAM Rac1 associated GEF 2 \\
\hline MED25 & ENSG00000104973 & Mediator complex subunit 25 \\
\hline $\mathrm{SHC} 1$ & ENSG00000160691 & SHC adaptor protein 1 \\
\hline RXRB & ENSG00000204231 & Retinoid $\mathrm{X}$ receptor beta \\
\hline FADS1 & ENSG00000149485 & Fatty acid desaturase 1 \\
\hline AHRR & ENSG00000063438 & Aryl-hydrocarbon receptor repressor \\
\hline MAPK3 & ENSG00000102882 & Mitogen-activated protein kinase 3 \\
\hline TXNRD1 & ENSG00000198431 & Thioredoxin reductase 1 \\
\hline MED7 & ENSG00000155868 & Mediator complex subunit 7 \\
\hline NPAS2 & ENSG00000170485 & Neuronal PAS domain protein 2 \\
\hline NCOR2 & ENSG00000196498 & Nuclear receptor corepressor 2 \\
\hline TGS1 & ENSG00000137574 & Trimethylguanosine synthase 1 \\
\hline NCOA6 & ENSG00000198646 & Nuclear receptor coactivator 6 \\
\hline NRF1 & ENSG00000106459 & Nuclear respiratory factor 1 \\
\hline MED24 & ENSG00000008838 & Mediator complex subunit 24 \\
\hline MED15 & ENSG00000099917 & Mediator complex subunit 15 \\
\hline MED20 & ENSG00000124641 & Mediator complex subunit 20 \\
\hline TNFRSF21 & ENSG00000146072 & TNF receptor superfamily member 21 \\
\hline PPARG & ENSG00000132170 & Peroxisome proliferator activated receptor gamma \\
\hline MED22 & ENSG00000148297 & Mediator complex subunit 22 \\
\hline MED12 & ENSG00000184634 & Mediator complex subunit 12 \\
\hline PDPK1 & ENSG00000140992 & 3-Phosphoinositide dependent protein kinase 1 \\
\hline CYP7A1 & ENSG00000167910 & Cytochrome P450 family 7 subfamily A member 1 \\
\hline ME1 & ENSG00000065833 & Malic enzyme 1 \\
\hline SIN3B & ENSG00000127511 & SIN3 transcription regulator family member B \\
\hline SREBF2 & ENSG00000198911 & Sterol regulatory element binding transcription factor 2 \\
\hline CD36 & ENSG00000135218 & CD36 molecule \\
\hline MED10 & ENSG00000133398 & Mediator complex subunit 10 \\
\hline NFYA & ENSG00000001167 & Nuclear transcription factor $Y$ subunit alpha \\
\hline CYP4A11 & ENSG00000187048 & Cytochrome P450 family 4 subfamily A member 11 \\
\hline PLIN2 & ENSG00000147872 & Perilipin 2 \\
\hline ACSL1 & ENSG00000151726 & Acyl-CoA synthetase long chain family member 1 \\
\hline GPS2 & ENSG00000132522 & G protein pathway suppressor 2 \\
\hline MED27 & ENSG00000160563 & Mediator complex subunit 27 \\
\hline SMARCD3 & ENSG00000082014 & $\begin{array}{l}\text { SWI/SNF related, matrix associated, actin depend- } \\
\text { ent regulator of chromatin, subfamily D, member } 3\end{array}$ \\
\hline
\end{tabular}

To confirm the applicability of our prognostic model, we performed stratified survival analysis according to gender, age, tumor grade, and stage, and. The prognostic model was applied to the risk score of patients in each group. The survival rate of patients in the highrisk and low-risk groups was significantly different in each group [Fig. 4c-f].

\section{Discussion}

HCC is the third cause of cancer-related deaths [17] and the sixth most common cancer worldwide, accounting for approximately $75-85 \%$ of all liver cancers [18]. In recent years, metabolic reprogramming is widely regarded as a hallmark of liver cancer associated with rapid cell proliferation $[19,20]$. Although the Warburg 
Table 211 mRNAs identified by univariate Cox analysis in HCC

\begin{tabular}{llllc}
\hline mRNA & HR & HR.95L & HR.95H & coxPvalue \\
\hline SHC1 & 1.4658 & 1.1424 & 1.8808 & 0.0026 \\
MAPK3 & 1.5682 & 1.1715 & 2.0993 & 0.0025 \\
NPAS2 & 1.5439 & 1.1773 & 2.0246 & 0.0017 \\
MED15 & 1.7047 & 1.2296 & 2.3634 & 0.0014 \\
MED20 & 1.4030 & 1.0303 & 1.9106 & 0.0316 \\
CYP4A11 & 0.9051 & 0.8287 & 0.9885 & 0.0266 \\
TNFRSF21 & 1.2054 & 1.0531 & 1.3798 & 0.0067 \\
PPARG & 1.2667 & 1.0569 & 1.5182 & 0.0105 \\
MED22 & 1.8974 & 1.3858 & 2.5979 & 0.0001 \\
ME1 & 1.2438 & 1.1009 & 1.4053 & 0.0005 \\
MED10 & 1.7496 & 1.3406 & 2.2835 & $<0.0001$ \\
\hline
\end{tabular}

effect and glutamine metabolism alterations are common processes in the metabolic reprogramming of tumors [21, 22]. Mounting evidence also suggests that lipid metabolic reprogramming is essential for HCC development [23]. For instance, HCC cells typically exhibit enhanced de novo synthesis of fatty acids [24]. Lipid metabolism alterations also play a critical role in the adaptation of cancer cells to hypoxic conditions and maintenance of cancer stem cells [25]. When it comes to tumors and metabolism, biomarkers flash in our minds. Although there are many studies on lipid metabolism reprogramming in liver cancer, studies linking lipid metabolism with liver cancer prognosis are still limited. In this way, we tried to find out a lipid metabolism-related biomarkers for $\mathrm{HCC}$ patients.

In this study, we performed various bioinformatics analyses and revealed the role of lipid metabolism-related genes in HCC patient prognosis. Specifically, we identified two lipid metabolism-related gene sets (totaling 163 genes) significantly enriched in HCC. Developed by David Cox [26], Cox regression is widely used to compare the survival of two or more groups and evaluate the effect of factors on survival. In this way, we identified 11 lipid metabolism-related genes that were associated with patient survival by performing univariate Cox regression analysis. Used LASSO regression analysis, were eliminated 8 functionally similar mRNAs. We used the remaining 3 mRNAs (ME1, MED10, and MED22) to construct a prognostic model.

The use of risk scores can facilitate a more accurate prediction of patient prognosis and guide clinical decision making [27]. An overwhelming number of genes have been identified as potential prognostic biomarkers for cancer [28-30]. Consequently, appropriate mathematical models for patient risk assessment are required. Here, we calculated a risk score for each patient based on the expression levels of $M E 1, M E D 10$, and $M E D 22$ and their corresponding coefficients. Survival analysis revealed a significant difference in the survival rate between the high-risk and low-risk groups. The risk curve constructed based on this prognostic model also confirmed that the expression of these three mRNAs and the number of deaths increased with increasing risk values. Similar analyses in a validation cohort confirmed the prognostic accuracy of this model. Univariate and multivariate Cox regression analyses indicated that the risk score and tumor stage were significant and independent prognostic factors in HCC. Stratified survival analysis showed that the survival rate of patients in the high-risk and low-risk group differed significantly in the different subgroups, highlighting the feasibility of our prognostic model.

ME1, MED10, and MED22 levels were found to be elevated in HCC tissues, both at the mRNA level and protein levels. ME1 is a multifunctional enzyme converting malate to pyruvate and is associated with lipid metabolism [31]. The beta-oxidation of fatty acid chains to produce energy requires acetyl-CoA [32]. In the meantime, a malic enzyme is needed for tricarboxylate shuttle so that acetyl-CoA can pass through the mitochondria into the cytoplasm [33]. The role of ME1 in cancer has previously been reported [34, 35]. It can induce epithelial-mesenchymal transition in a ROS (reactive oxygen species)dependent manner [36]. MED10 and MED22 are both subunits of the Mediator complex, an evolutionary conserved multiprotein complex consisting of approximately 30 subunits. The Mediator complex functions as a transcriptional coactivator in eukaryotes $[37,38]$, regulating the expression of most RNA polymerase II-transcribed genes [39]. Understanding the function of the Mediator complex in cancer is critical, as it may guide the development of novel anti-cancer therapies [40]. However, the role of the Mediator complex in HCC merits further investigation.

Epigenetic modifiers are promising targets for the treatment of HCC, as they play an essential role in regulating HCC cell proliferation and metastasis [41]. DNA methylation is a critical epigenetic regulation mechanism, playing a vital role in tumor development by altering the expression of various tumor-associated genes [42]. Gene mutations are key to tumor development and progression [43]. In this study, we identified varying degrees of mutations in $M E 1, M E D 10$, and MED22 in HCC patients. We also found that their expression levels were negatively correlated with their gene methylation levels. These data suggest that the mutations and aberrant gene methylation in ME1, MED10, and MED22 in HCC tissues might contribute to their upregulation in HCC. Therefore, a more in-depth understanding of the epigenetic changes in these genes may facilitate the development of targeted therapies for HCC. 

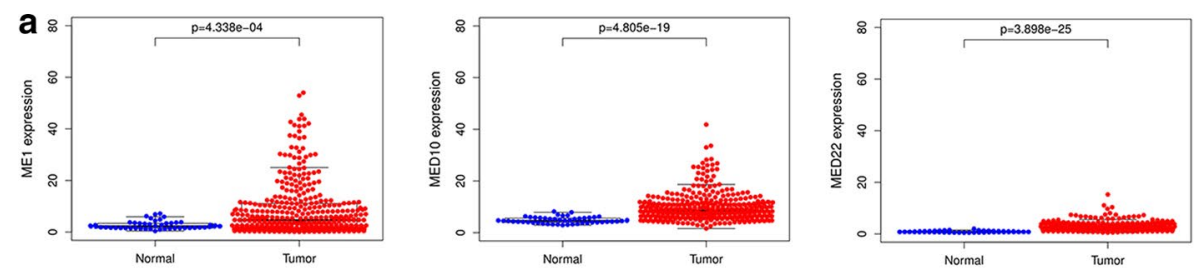

b

ME1 Normal

ME1 HCC
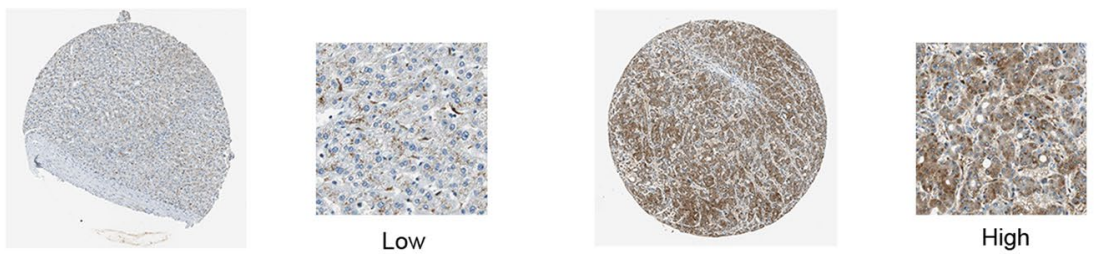

MED22 Normal

MED22 HCC
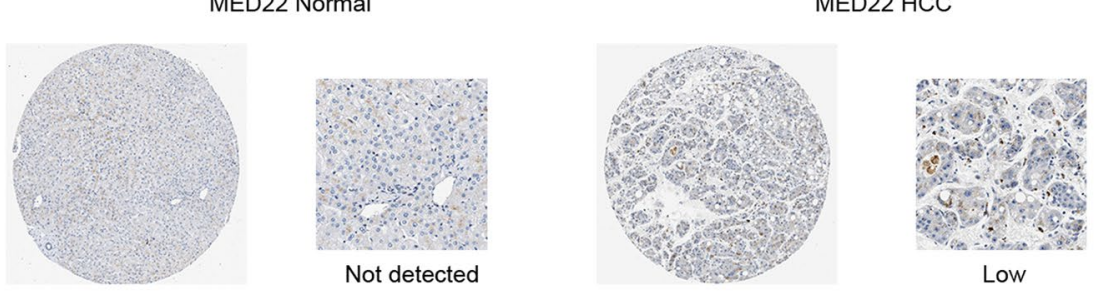

C
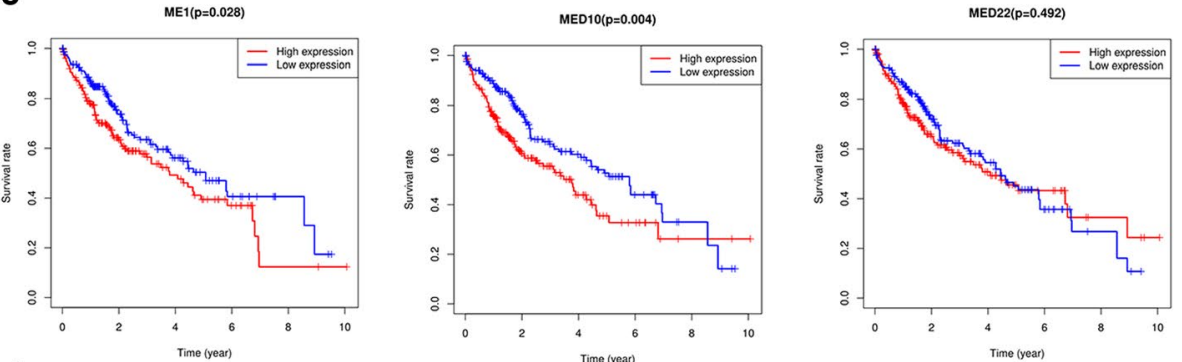

d
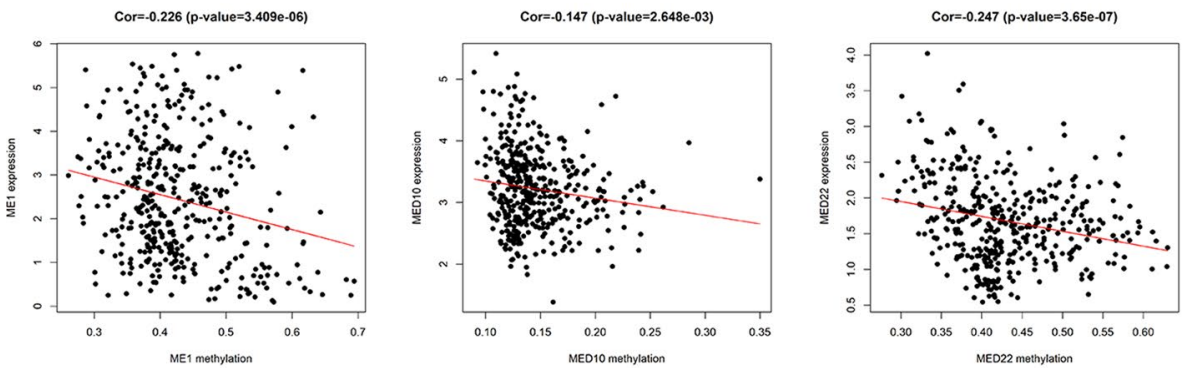

e

PPARg

ме222

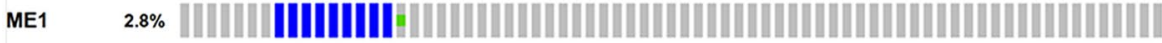
м.210 $\quad$ 1.7\%

Genetic Alteration

Fig. 2 The expression level and epigenetic modification of three genes in HCC. a Differential expression analysis of three genes in mRNA level. $\mathbf{b}$ Representative immunohistochemistry staining of three proteins in normal tissue and HCC tissue from The Human Protein Atlas. c K-M analysis of three mRNAs. $\mathbf{d}$ Correlation between gene expression level and methylation degree. e Mutations of three genes in HCC 

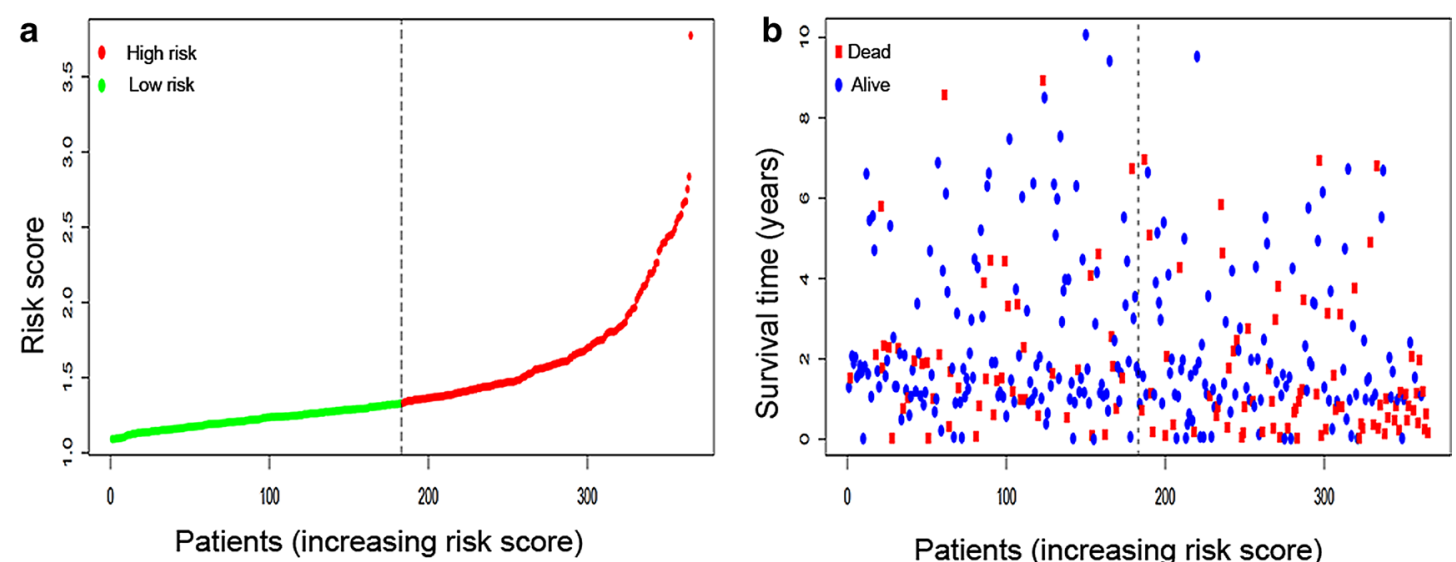

Patients (increasing risk score)
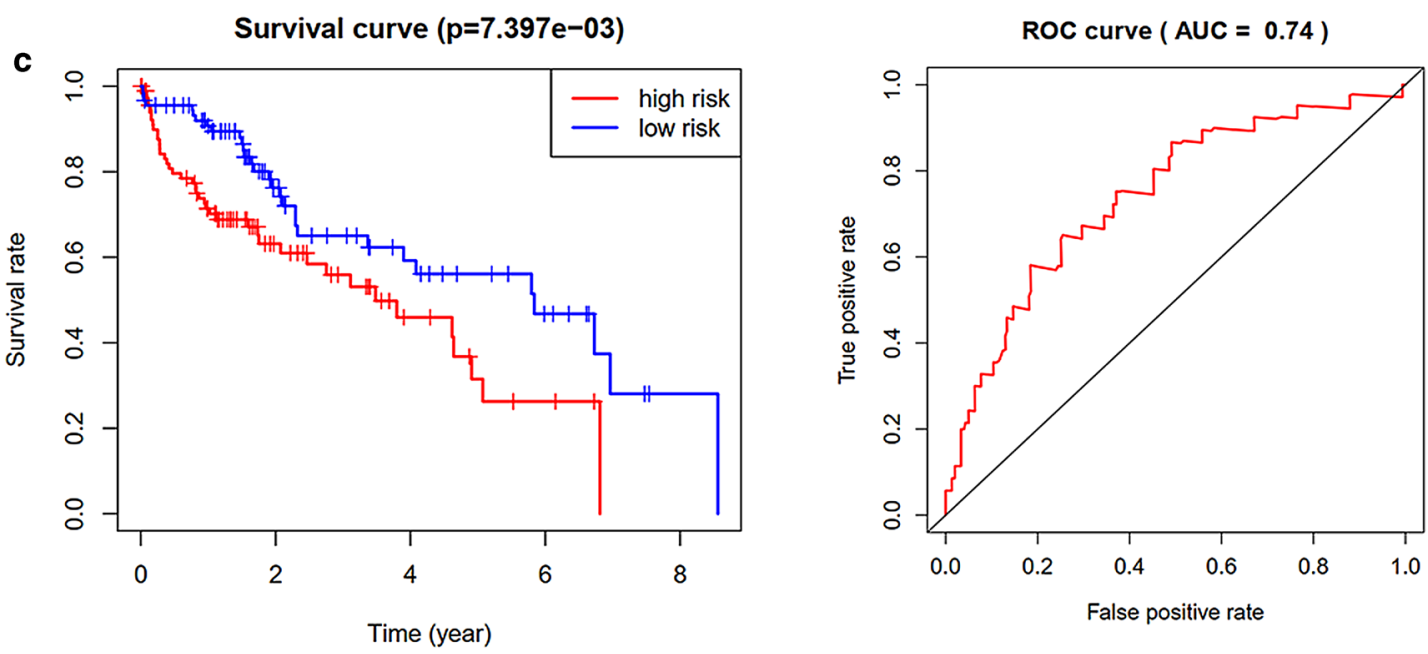

Survival curve $(p=1.576 \mathrm{e}-06)$

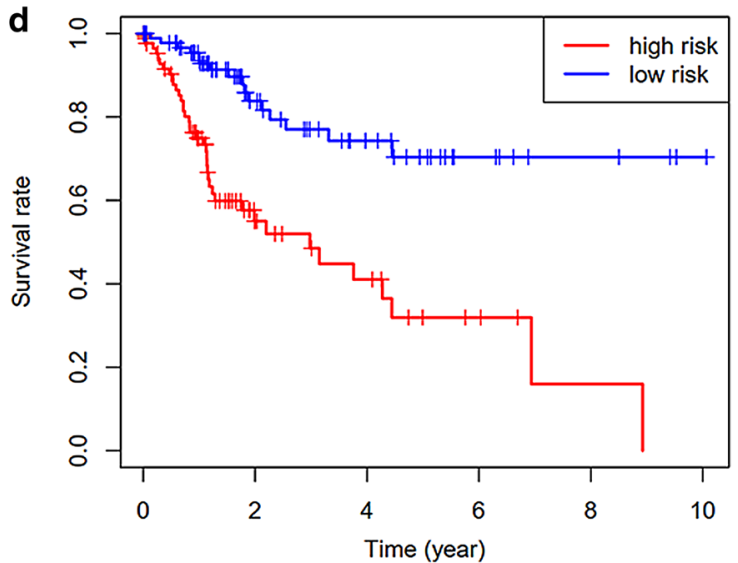

ROC curve $($ AUC $=0.762)$

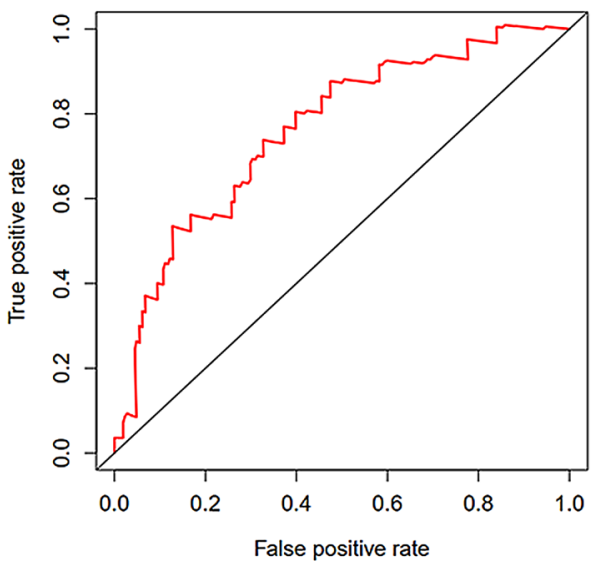

Fig. 3 Construction and validation of a three mRNAs prognostic model. a HCC patients were divided into high-risk and low-risk groups according to their median risk scores. $\mathbf{b}$ Survival time and survival status of patients changed along with the value of risk score. $\mathbf{c ~ K - M}$ analysis and ROC curve for training set. $\mathbf{d}$ K-M analysis and ROC curve for validation set 
Table 3 Clinical pathological parameters of patients and the chi-square test of the relation between clinical features and risk score

\begin{tabular}{|c|c|c|c|c|c|}
\hline \multirow[t]{2}{*}{ Clinical feature } & \multirow[t]{2}{*}{$\mathrm{N}$} & \multicolumn{2}{|l|}{ Risk score } & \multirow[t]{2}{*}{$x^{2}$} & \multirow[t]{2}{*}{$p$} \\
\hline & & High risk n(\%) & Low risk n(\%) & & \\
\hline Age & & & & 66.60 & $<0.001$ \\
\hline$>65$ & 276 & $65(23.55 \%)$ & $211(76.45 \%)$ & & \\
\hline$\leq 65$ & 192 & $117(60.94 \%)$ & $75(39.06 \%)$ & & \\
\hline Gender & & & & 0.38 & 0.54 \\
\hline Male & 309 & $123(39.81 \%)$ & $186(60.19 \%)$ & & \\
\hline Female & 160 & $59(36.88 \%)$ & $101(63.13 \%)$ & & \\
\hline Child pugh classification grade & & & & 2.41 & 0.12 \\
\hline A & 271 & $103(38.01 \%)$ & $168(61.99 \%)$ & & \\
\hline$B-C$ & 33 & $8(24.24 \%)$ & $25(75.76 \%)$ & & \\
\hline Adjacent hepatic tissue inflammation & & & & 0.00 & 0.98 \\
\hline Yes & 146 & $48(32.88 \%)$ & $98(67.12 \%)$ & & \\
\hline No & 162 & $53(32.72 \%)$ & 109 (67.28\%) & & \\
\hline BMI & & & & 1.95 & 0.16 \\
\hline$\geq 30$ & 93 & $31(33.33 \%)$ & $62(66.67 \%)$ & & \\
\hline$<30$ & 324 & $134(41.36 \%)$ & 190 (58.64\%) & & \\
\hline Cirrhosis & & & & 0.23 & 0.63 \\
\hline Yes & 93 & $27(29.03 \%)$ & $66(70.97 \%)$ & & \\
\hline No & 119 & $31(26.05 \%)$ & $88(73.95 \%)$ & & \\
\hline Grade & & & & 16.73 & $<0.001$ \\
\hline$|-| \mid$ & 295 & $94(31.86 \%)$ & 201 (68.14\%) & & \\
\hline III-IV & 166 & $85(51.20 \%)$ & $81(48.80 \%)$ & & \\
\hline Stage & & & & 2.60 & 0.11 \\
\hline$|-| \mid$ & 306 & $118(38.56 \%)$ & $188(61.44 \%)$ & & \\
\hline III-IV & 112 & $53(47.32 \%)$ & $59(52.68 \%)$ & & \\
\hline Barcelona clinic liver cancer & & & & 0.14 & 0.71 \\
\hline$A-B$ & 370 & $144(38.92 \%)$ & $226(61.08 \%)$ & & \\
\hline$C-D$ & 31 & $11(35.48 \%)$ & $20(64.52 \%)$ & & \\
\hline Neoadjuvant treatment & & & & 1.27 & 0.26 \\
\hline Yes & 2 & $0(0.00 \%)$ & $2(100.00 \%)$ & & \\
\hline No & 467 & $182(38.97 \%)$ & $285(61.03 \%)$ & & \\
\hline Radiation therapy & & & & 0.14 & 0.71 \\
\hline Yes & 9 & $3(33.33 \%)$ & $6(66.67 \%)$ & & \\
\hline No & 372 & 147 (39.52\%) & $225(60.48 \%)$ & & \\
\hline Person neoplasm cancer status & & & & 1.32 & 0.25 \\
\hline Tumor free & 195 & $70(35.90 \%)$ & $125(64.10 \%)$ & & \\
\hline With tumor & 165 & $69(41.82 \%)$ & $96(58.18 \%)$ & & \\
\hline Relative family cancer history & & & & 6.51 & 0.01 \\
\hline Yes & 162 & $49(30.25 \%)$ & $113(69.75 \%)$ & & \\
\hline No & 248 & $106(42.74 \%)$ & $142(57.26 \%)$ & & \\
\hline
\end{tabular}

\section{Conclusions}

We identified three lipid-metabolism-related genes (ME1, MED10, and MED22) that are upregulated in HCC tissues, and established a 3-gene signature-based risk model that can accurately predict HCC patient prognosis. Notably, HCC patients with high-risk scores are more likely to experience worse outcomes than lowrisk patients. We also provide preliminary data on the possible mechanisms underlying the pro-tumorigenic effects of these genes in HCC. The findings of this study support the strong link between lipid metabolism and HCC progression and may guide the development of 


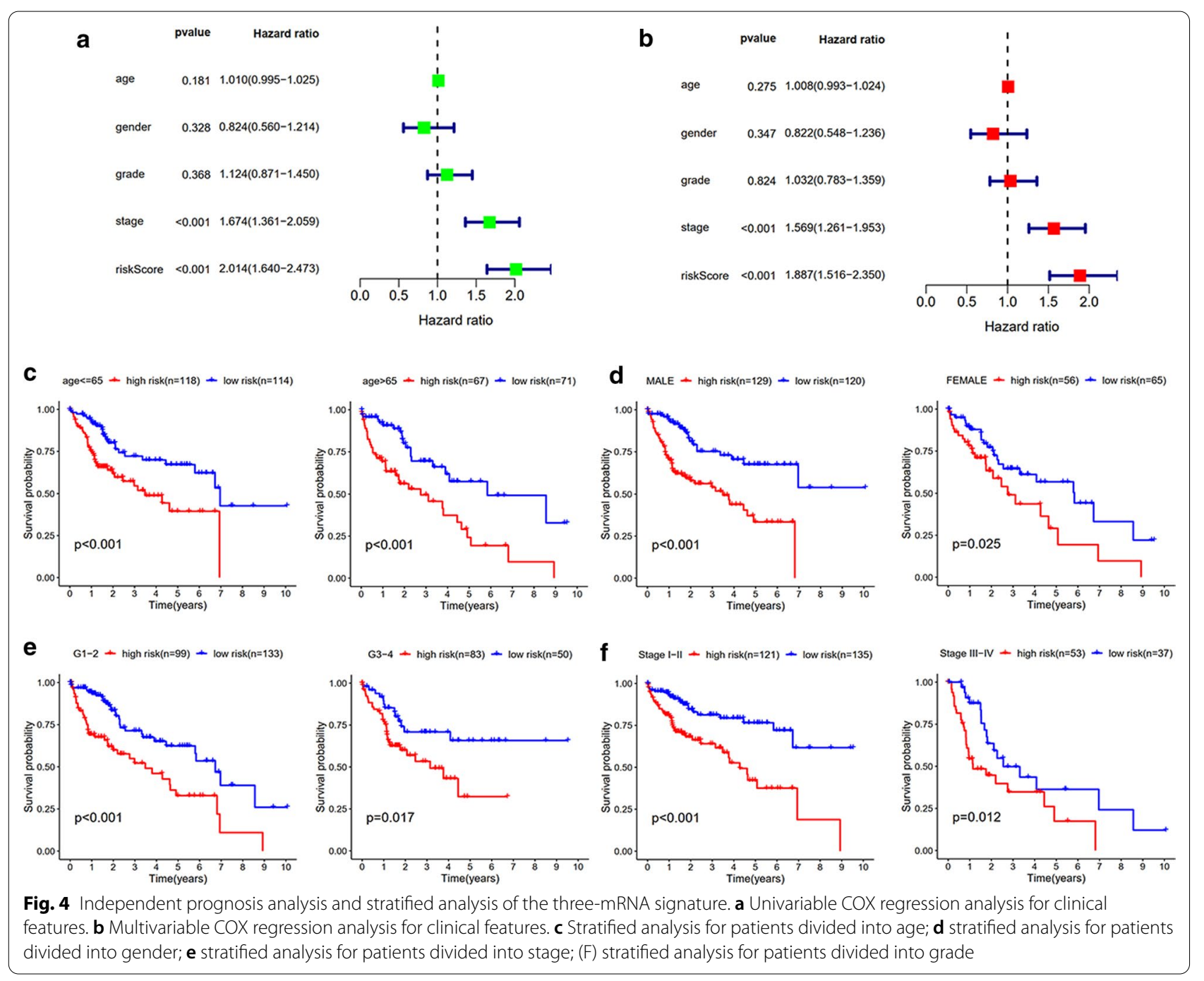

novel metabolism-targeted treatment approaches for HCC.

\section{Abbreviations}

HCC: Hepatocellular carcinoma; TCGA: The Cancer Genome Atlas; GSEA: Gene set enrichment analysis; ROC: Receiver operating characteristic; LASSO: Least absolute shrinkage and selection operator; AUC: Area under the curve; GO: Gene ontology; DAVID: The Database for Annotation, Visualization, and Integrated Discovery; ROS: Reactive oxygen species.

\section{Acknowledgements}

Not applicable.

\section{Authors' contributions}

Study conception and design: WW, CZ, ZS. Acquisition of data: CZ, QY, XY. Statistical analysis: XZ, CY, GL. Analysis and interpretation of data: WW, CZ, QY, XY. Drafting of manuscript: WW. Critical revision: WW, QY, GL, ZS. Guarantor of article: ZS. All authors read and approved the final manuscript.

\section{Funding}

This work was funded by the grants from the National Natural Science Foundation of China (No. 91959107).

\section{Availability of data and materials}

The gene expression datasets, gene methylation profiles, and clinicopathological characteristics of HCC patients were obtained from the cancer genome atlas database (https://tcga-data.nci.nih.gov/tcga/). The immunohistochemistry staining data was obtained from the human protein atlas database (https ://www.proteinatlas.org/). Gene mutations in HCC patients were obtained from cBioPortal database (https://www.cbioportal.org/). Detailed information about 35 mRNAs in Table 1 was obtained from the human gene database (https://www.genecards.org/).

\section{Ethics approval and consent to participate}

Not applicable.

\section{Consent for publication}

Not applicable.

\section{Competing interests}

The authors declare that they have no competing interests.

\section{Author details}

${ }^{1}$ Department of Hepatobiliary Surgery, Union Hospital, Tongji Medical College, Huazhong University of Science and Technology, 1277 Jiefang Avenue, Wuhan 430022, China. ${ }^{2}$ Hepatic Surgery Center, Tongji Hospital, 
Tongji Medical College, Huazhong University of Science and Technology, 1095 Jiefang Avenue, Wuhan 430030, China. ${ }^{3}$ Clinical Medical Research Center of Hepatic Surgery at Hubei Province, Wuhan, China. ${ }^{4}$ School of Life Science and Technology, Huazhong University of Science and Technology, Wuhan 430074, China.

Received: 3 September 2020 Revised: 28 January 2021 Accepted: 2 February 2021

Published online: 12 February 2021

\section{References}

1. Kulik L, El-Serag HB. Epidemiology and management of hepatocellular carcinoma. Gastroenterology. 2019;156(2):477-91.e1.

2. Llovet JM, Zucman-Rossi J, Pikarsky E, et al. Hepatocellular carcinoma. Nat Rev Dis Primers. 2016;2:16018.

3. Raza A, Sood GK. Hepatocellular carcinoma review: current treatment, and evidence-based medicine. World J Gastroenterol. 2014;20(15):4115-27.

4. Lin H, Fan X, He L, et al. Methylation patterns of RASA3 associated with clinicopathological factors in hepatocellular carcinoma. J Cancer 2018;9(12):2116-22.

5. Torre LA, Bray F, Siegel RL, et al. Global cancer statistics, 2012. CA Cancer J Clin. 2015;65(2):87-108.

6. Zhao L, Jiang L, He L, et al. Identification of a novel cell cycle-related gene signature predicting survival in patients with gastric cancer. J Cell Physiol. 2019;234(5):6350-60.

7. Benjamin DI, Cravatt BF, Nomura DK. Global profiling strategies for mapping dysregulated metabolic pathways in cancer. Cell Metab. 2012;16(5):565-77.

8. Boroughs LK, DeBerardinis RJ. Metabolic pathways promoting cancer cell survival and growth. Nat Cell Biol. 2015;17(4):351-9.

9. Liu MX, Jin L, Sun SJ, et al. Metabolic reprogramming by PCK1 promotes TCA cataplerosis, oxidative stress and apoptosis in liver cancer cells and suppresses hepatocellular carcinoma. Oncogene. 2018;37(12):1637-53.

10. Zhao W, Guan J, Horswell R, et al. HDL cholesterol and cancer risk among patients with type 2 diabetes. Diabetes Care. 2014;37(12):3196-203.

11. Pope ED 3rd, Kimbrough EO, Vemireddy LP, et al. Aberrant lipid metabolism as a therapeutic target in liver cancer. Expert Opin Ther Targets. 2019;23(6):473-83.

12. Cooper LA, Demicco EG, Saltz JH, et al. PanCancer insights from The Cancer Genome Atlas: the pathologist's perspective. J Pathol. 2018;244(5):512-24

13. Jiao XD, Qin BD, You P, et al. The prognostic value of TP53 and its correlation with EGFR mutation in advanced non-small cell lung cancer, an analysis based on cBioPortal data base. Lung Cancer. 2018;123:70-5.

14. Subramanian A, Tamayo P, Mootha VK, et al. Gene set enrichment analysis: a knowledge-based approach for interpreting genome-wide expression profiles. Proc Natl Acad Sci U S A. 2005:102(43):15545-50.

15. Li T, Gao X, Han L, Yu J, Li H. Identification of hub genes with prognostic values in gastric cancer by bioinformatics analysis. World J Surg Oncol. 2018;16(1):114

16. Mayr A, Hofner B, Schmid M. Boosting the discriminatory power of sparse survival models via optimization of the concordance index and stability selection. BMC Bioinform. 2016;17:288.

17. Bray F, Ferlay J, Soerjomataram I, et al. Global cancer statistics 2018: GLOBOCAN estimates of incidence and mortality worldwide for 36 cancers in 185 countries. CA Cancer J Clin. 2018;68(6):394-424.

18. Ferlay J, Soerjomataram I, Dikshit R, et al. Cancer incidence and mortality worldwide: sources, methods and major patterns in GLOBOCAN 2012. Int J Cancer. 2015;136(5):E359-86.

19. Ward PS, Thompson CB. Metabolic reprogramming: a cancer hallmark even warburg did not anticipate. Cancer Cell. 2012;21(3):297-308.

20. DeBerardinis RJ, Lum JJ, Hatzivassiliou G, et al. The biology of cancer: metabolic reprogramming fuels cell growth and proliferation. Cell Metab. 2008;7(1):11-20

21. Hsu PP, Sabatini DM. Cancer cell metabolism: Warburg and beyond. Cell. 2008;134(5):703-7.
22. Cairns RA, Harris IS, MakTW. Regulation of cancer cell metabolism. Nat Rev Cancer. 2011;11(2):85-95.

23. Sangineto M, Villani R, Cavallone F, Romano A, Loizzi D, Serviddio G. Lipid metabolism in development and progression of hepatocellular carcinoma. Cancers (Basel). 2020;12(6):1419.

24. Budhu A, Roessler S, Zhao X, et al. Integrated metabolite and gene expression profiles identify lipid biomarkers associated with progression of hepatocellular carcinoma and patient outcomes. Gastroenterology. 2013;144(5):1066-75.e1.

25. Nakagawa H, Hayata Y, Kawamura S, Yamada T, Fujiwara N, Koike K. Lipid metabolic reprogramming in hepatocellular carcinoma. Cancers (Basel). 2018;10(11):447.

26. Cox DR. Regression models and life-tables. J R Stat Soc Ser B (Methodol). 1972:34:187-220.

27. Liu L, Hu J, Wang Y, et al. Establishment of a novel risk score model by comprehensively analyzing the immunogen database of bladder cancer to indicate clinical significance and predict prognosis. Aging. 2020;12(12):11967-89.

28. Barrón EV, Roman-Bassaure E, Sánchez-Sandoval AL, et al. CDKN3 mRNA as a biomarker for survival and therapeutic target in cervical cancer. PLoS One. 2015;10(9):e0137397.

29. Waligórska-Stachura J, Sawicka-Gutaj N, Zabel M, et al. Survivin DEx3 as a biomarker of thyroid cancers: a study at the mRNA and protein level. Oncol Lett. 2017;13(4):2437-41.

30. Li Z, Li F, Peng Y, Fang J, Zhou J. Identification of three m6A-related mRNAs signature and risk score for the prognostication of hepatocellular carcinoma. Cancer Med. 2020:9(5):1877-89.

31. Rice CM, Davies LC, Subleski JJ, et al. Tumour-elicited neutrophils engage mitochondrial metabolism to circumvent nutrient limitations and maintain immune suppression. Nat Commun. 2018;9(1):5099.

32. Babrak L, Danelishvili L, Rose SJ, Kornberg T, Bermudez LE. The environment of "Mycobacterium avium subsp. hominissuis" microaggregates induces synthesis of small proteins associated with efficient infection of respiratory epithelial cells. Infect Immun. 2015;83(2):625-36.

33. Vidal O, Varona L, Oliver MA, Noguera JL, Sànchez A, Amills M. Malic enzyme 1 genotype is associated with backfat thickness and meat quality traits in pigs. Anim Genet. 2006;37(1):28-32.

34. Liao R, Ren G, Liu H, et al. ME1 promotes basal-like breast cancer progression and associates with poor prognosis. Sci Rep. 2018;8(1):16743.

35. Wen D, Liu D, Tang J, et al. Malic enzyme 1 induces epithelial-mesenchymal transition and indicates poor prognosis in hepatocellular carcinoma. Tumour Biol. 2015:36(8):6211-21.

36. Quevedo M, Meert L, Dekker MR, et al. Mediator complex interaction partners organize the transcriptional network that defines neural stem cells. Nat Commun. 2019;10(1):2669.

37. Kim YJ, Björklund S, Li Y, Sayre MH, Kornberg RD. A multiprotein mediator of transcriptional activation and its interaction with the C-terminal repeat domain of RNA polymerase II. Cell. 1994;77(4):599-608.

38. Malik S, Roeder RG. The metazoan mediator co-activator complex as an integrative hub for transcriptional regulation. Nat Rev Genet. 2010;11(11):761-72.

39. Weber H, Garabedian MJ. The mediator complex in genomic and nongenomic signaling in cancer. Steroids. 2018;133:8-14.

40. Brägelmann J, Klümper N, Offermann A, et al. Pan-cancer analysis of the mediator complex transcriptome identifies CDK19 and CDK8 as therapeutic targets in advanced prostate cancer. Clin Cancer Res. 2017;23(7):1829-40.

41. Han TS, Ban HS, Hur K, Cho HS. The epigenetic regulation of HCC metastasis. Int J Mol Sci. 2018;19(12):3978.

42. Mahmoud AM, Ali MM. Methyl donor micronutrients that modify DNA methylation and cancer outcome. Nutrients. 2019;11(3):608.

43. Horn HF, Vousden $\mathrm{KH}$. Coping with stress: multiple ways to activate $\mathrm{p} 53$. Oncogene. 2007;26(9):1306-16

\section{Publisher's note}

Springer Nature remains neutral with regard to jurisdictional claims in published maps and institutional affiliations. 